\title{
THE ALHAMBRA PHOTOMETRIC SYSTEM
}

\author{
T. Aparicio Villegas ${ }^{1}$, E. J. Alfaro ${ }^{1}$, J. Cabrera-Caño ${ }^{1,2}$, M. Moles ${ }^{1,3}$, N. Benítez ${ }^{1}$, J. Perea ${ }^{1}$, A. del Olmo $^{1}$, \\ A. Fernández-Soto ${ }^{4}$, D. Cristóbal-Hornillos ${ }^{1,3}$, C. Husillos $^{1}$, J. A. L. Aguerri ${ }^{5}$, T. Broadhurst ${ }^{6}$, F. J. Castander ${ }^{7}$, \\ J. Cepa ${ }^{5,8}$, M. Cerviño ${ }^{1}$, R. M. González Delgado ${ }^{1}$, L. Infante ${ }^{9}$, I. Márquez ${ }^{1}$, J. Masegosa ${ }^{1}$, V. J. Martínez ${ }^{10,11}$, \\ F. Prada ${ }^{1}$, J. M. Quintana ${ }^{1}$, And S. F. SÁNChez ${ }^{3,12}$ \\ ${ }^{1}$ Instituto de Astrofísica de Andalucía (CSIC), E-18080, Granada, Spain; terenz@iaa.es, emilio@iaa.es, benitez@iaa.es, jaime@iaa.es, chony@iaa.es, cesar@iaa.es, \\ mcs@iaa.es, rosa@iaa.es, isabel@iaa.es,pepa@iaa.es, fprada@iaa.es, quintana@iaa.es \\ ${ }^{2}$ Facultad de Física, Departamento de Física Atómica, Molecular y Nuclear, Universidad de Sevilla, Sevilla, Spain; jcc-famn@us.es \\ ${ }^{3}$ Centro de Estudios de Física del Cosmos de Aragón (CEFCA), C/General Pizarro, 1, 44001 Teruel, Spain; moles@cefca.es, dch@cefca.es, sanchez@cefca.es \\ ${ }^{4}$ Instituto de Física de Cantabria (CSIC-UC), 39005, Santander, Spain; fsoto@ ifca.unican.es \\ ${ }^{5}$ Instituto de Astrofísica de Canarias, La Laguna, Tenerife, Spain; jalfonso@ iac.es \\ ${ }^{6}$ School of Physics and Astronomy, Tel Aviv University, Israel; tjb@wise.tau.ac.il \\ ${ }^{7}$ Institut de Ciències de l'Espai, IEEC-CSIC, Barcelona, Spain; fjc@ieec.fcr.es \\ ${ }^{8}$ Departamento de Astrofísica, Facultad de Física, Universidad de la Laguna, Spain; jcn@iac.es \\ ${ }^{9}$ Departamento de Astronomía, Pontificia Universidad Católica, Santiago, Chile; linfante@ astro.puc.cl \\ ${ }^{10}$ Departament d'Astronomía i Astrofísica, Universitat de València, Valencia, Spain; vicent.martinez@uv.es \\ ${ }^{11}$ Observatori Astronòmic de la Universitat de València, Valencia, Spain \\ ${ }^{12}$ Centro Astronómico Hispano-Alemán, Almería, Spain \\ Received 2009 August 14; accepted 2010 January 11; published 2010 February 11
}

\begin{abstract}
This paper presents the characterization of the optical range of the ALHAMBRA photometric system, a 20 contiguous, equal-width, medium-band CCD system with wavelength coverage from $3500 \AA$ to $9700 \AA$. The photometric description of the system is done by presenting the full response curve as a product of the filters, CCD, and atmospheric transmission curves, and using some first- and second-order moments of this response function. We also introduce the set of standard stars that defines the system, formed by 31 classic spectrophotometric standard stars which have been used in the calibration of other known photometric systems, and 288 stars, flux calibrated homogeneously, from the Next Generation Spectral Library (NGSL). Based on the NGSL, we determine the transformation equations between Sloan Digital Sky Survey ugriz photometry and the ALHAMBRA photometric system, in order to establish some relations between both systems. Finally, we develop and discuss a strategy to calculate the photometric zero points of the different pointings in the ALHAMBRA project.
\end{abstract}

Key words: cosmology: observations - galaxies: distances and redshifts - instrumentation: photometers standards - stars: fundamental parameters - techniques: photometric

Online-only material: machine-readable and VO tables

\section{INTRODUCTION}

The ALHAMBRA survey is a project aimed at getting a photometric data super-cube, which samples a cosmological fraction of the universe with enough precision to draw an evolutionary track of its content and properties (see Moles et al. 2008 for a more detailed description of the scientific objectives of the project). The global strategy to achieve this aim is to identify a large number of objects, families, or structures at different redshifts $(z)$, and compare their properties as a function of $z$, once the physical dispersion of those properties at every $z$-value has been taken into account. Thus, it is possible to identify epochs (measured by $z$ ) of development of some families of objects or structures, or their disappearance, or even the progressive change in the proportion of a family of objects and the degree of structuring of the universe. Such a survey requires a combination of large area, depth, photometric accuracy, and spectral coverage. The number, width, and position of the filters composing an optimal filter set to accomplish these objectives have been discussed and evaluated by Benítez et al. (2009), yielding a filter set formed by a uniform system of 20 constant-width, non-overlapping, medium-band filters in the optical range plus the three standard $J H K_{s}$ near-infrared (NIR) bands.
The ALHAMBRA project aims at covering a minimum of $4 \mathrm{deg}^{2}$ in eight discontinuous regions of the sky. This implies the need for a wide-field instrument, associated with a 3-4 m telescope that permits coverage of the selected area with the required signal-to-noise ratio, within a reasonable observing time period. The ALHAMBRA optical system was thus incorporated on the wide-field camera Large Area Imager for Calar Alto ${ }^{13}$ (LAICA) placed at the prime focus of the $3.5 \mathrm{~m}$ telescope at the Centro Astronómico Hispano Alemán (CAHA) at Calar Alto Observatory, while the NIR range was observed with the camera OMEGA-2000 ${ }^{14}$ attached to the same telescope.

The main purpose of this paper is to present the characterization of the 20 ALHAMBRA filters in the optical range, which covers from $3500 \AA$ to $9700 \AA$ at intervals of approximately $310 \AA$, to select the best set of standard stars that determine the observational system, to establish the first transformation equations between ALHAMBRA and other photometric systems already defined, and to check the strategies for the determination of the zero points in the automatic reduction of the ALHAMBRA data set. Some of these issues have been briefly

\footnotetext{
13 http://www.caha.es/CAHA/Instruments/LAICA/index.html

14 http://www.caha.es/CAHA/Instruments/O2000/index.html
} 
presented in a previous paper introducing the ALHAMBRA project (Moles et al. 2008).

In Section 2, we present the definition of the photometric system. The set of ALHAMBRA photometric standard stars is analyzed in detail in Section 3. In Section 4.1, transformation equations between the Sloan Digital Sky Survey (SDSS) and the ALHAMBRA photometric system are established from the comparison between the synthetic magnitudes of 288 stars from the Next Generation Spectral Library (NGSL) in both systems. We present color data for three galaxy templates as a function of redshift using the ALHAMBRA photometric system in Section 4.2 and in Section 4.3 we discuss the validity of the transformation equations for galaxies.

Finally, in Section 5, we develop a strategy to determine the photometric zero points of the system with uncertainties below a few hundredths of magnitude, to be applied in the calibration of the eight ALHAMBRA fields.

\section{CHARACTERIZATION OF THE ALHAMBRA PHOTOMETRIC SYSTEM}

The ALHAMBRA photometric system has been defined according to what is specified in Benítez et al. (2009), as a system of 20 constant-width, non-overlapping, medium-band filters in the optical range (with wavelength coverage from $3500 \AA$ to $9700 \AA$ ). The definition of the system is given by the response curve defined by the product of three different transmission curves: the filter set, detector, and atmospheric extinction at 1.2 air masses, based on the CAHA monochromatic extinction tables (Sánchez et al. 2007). The graphic representation of the optical ALHAMBRA photometric system is shown in Figure 1. Numerical values of these response curves can be found at the Web page http://alhambra.iaa.es:8080.

First- and second-order moments of the transmission functions of the 20 different filters are also of interest. We will characterize the system filters using their isophotal wavelengths, full width half-maxima, and other derived quantities as follows.

Let $E_{\lambda}$ be the spectral energy distribution (SED) of an astronomical source and $S_{\lambda}$ the response curve of a filter in the photometric system defined as

$$
S_{\lambda}=T_{t}(\lambda) T_{f}(\lambda) T_{a}(\lambda),
$$

where $T_{t}$ is the product of the throughput of the telescope, instrument, and quantum efficiency of the detector; $T_{f}$ is the filter transmission; and $T_{a}$ is the atmospheric transmission. Following the definition in Golay (1974), assuming that the function $E_{\lambda}$ is continuous and that $S_{\lambda}$ does not change sign in a wavelength interval $\lambda_{a}-\lambda_{b}$, the mean value theorem states that there is at least one $\lambda_{i}$ inside the interval $\lambda_{a}-\lambda_{b}$, such that

$$
E_{\lambda_{i}} \int_{\lambda_{a}}^{\lambda_{b}} S_{\lambda} d \lambda=\int_{\lambda_{a}}^{\lambda_{b}} E_{\lambda} S_{\lambda} d \lambda
$$

implying,

$$
E_{\lambda_{i}}=\left\langle E_{\lambda}\right\rangle=\frac{\int_{\lambda_{a}}^{\lambda_{b}} E_{\lambda} S_{\lambda} d \lambda}{\int_{\lambda_{a}}^{\lambda_{b}} S_{\lambda} d \lambda},
$$

where $\lambda_{i}$ is the isophotal wavelength and $\left\langle E_{\lambda}\right\rangle$ denotes the mean value of the intrinsic flux above the atmosphere over the wavelength interval (see also Tokunaga \& Vacca 2005). Table 1 shows the isophotal wavelengths of the ALHAMBRA filters using the spectrum of VEGA as a reference. The absolute calibrated spectrum has been taken from the STScI Observatory Support
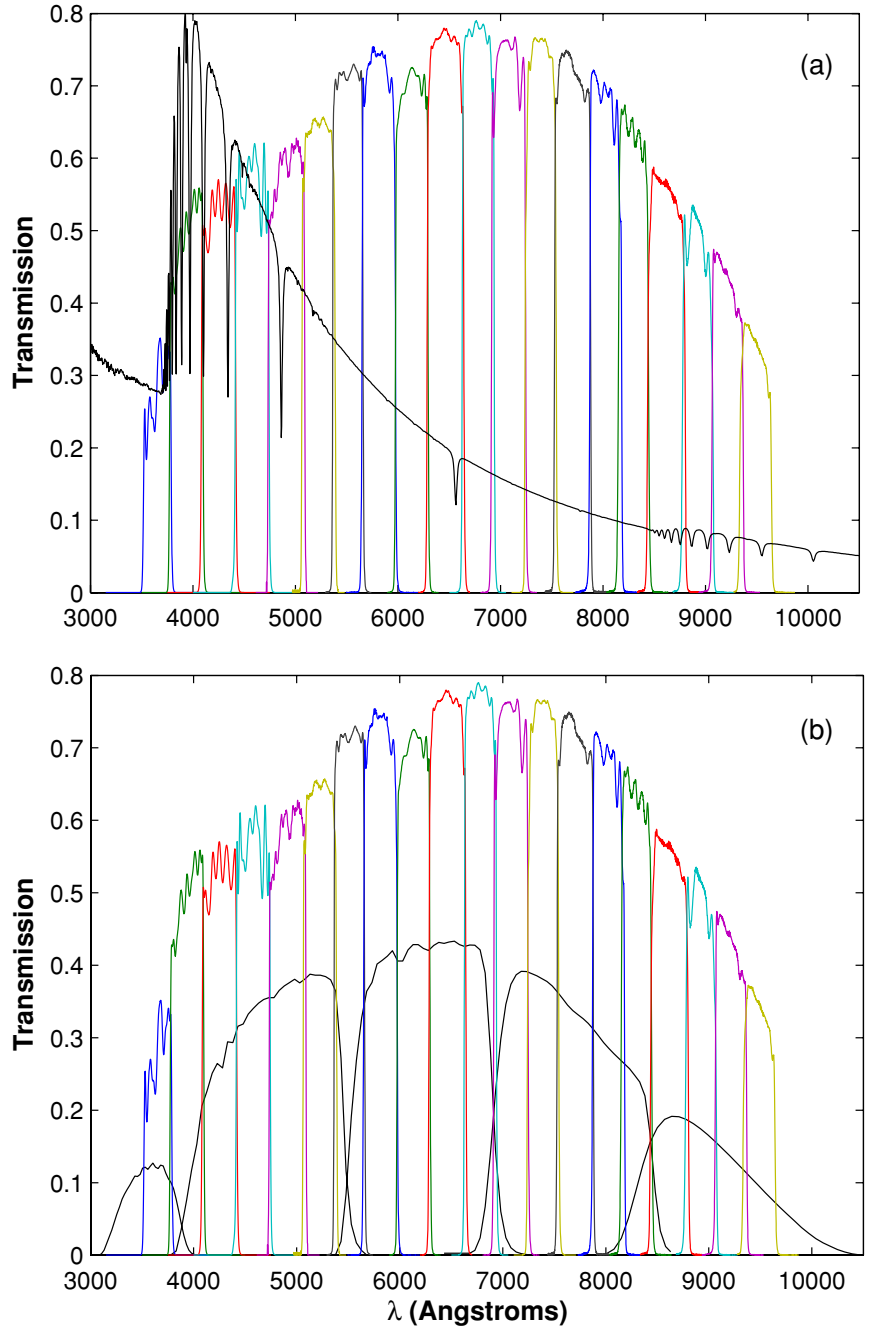

Figure 1. Response functions of the ALHAMBRA photometric system filters including atmospheric transmission at 1.2 air masses at the altitude of Calar Alto Observatory. Panel (a) also represents the spectrum of VEGA superimposed on the transmission curves. The flux of VEGA has been normalized and scaled properly to make the graphic possible. Panel (b) shows the response curves of the SDSS standard system (USNO) and the ALHAMBRA photometric system.

Group Web site, ${ }^{15}$ as the file alpha_lyr_stis_004.fits. This spectrum is a combination of modeled and observed fluxes consisting of Space Telescope Imaging Spectrograph (STIS) CCD fluxes from $3500 \AA$ to $5300 \AA$, and a Kurucz model with $T_{\text {eff }}=9400 \mathrm{~K}$ (Kurucz 2005) from $5300 \AA$ to the end of the spectral range. The isophotal wavelengths were calculated using Equation (3).

The determination of the isophotal wavelengths of a star in a photometric system can be complex, specially for the filters that contain conspicuous stellar absorption lines (see Figure 1(a)). For example, filter A394M is extremely sensitive to the Balmer jump and filter A425M encompasses the two prominent $H_{\gamma}$ and $H_{\delta}$ lines, at $4340 \AA$ and $4101 \AA$, respectively. At $4861 \AA, H_{\beta}$ is in filter A491M, and at $6563 \AA H_{\alpha}$ falls on filter A646M. The Paschen series, from 8208 Aat filter A829M, appears associated to the four reddest ALHAMBRA filters (A861M, A892M, A921M, A948M).

However, this quantity depends on the SED of the emitter; thus, for the same filter it will be different for each kind of source. The isophotal wavelength can be approximated by other central

\footnotetext{
15 http://www.stsci.edu/hst/observatory/cdbs/calspec.html
} 
Table 1

Representative Parameters of the ALHAMBRA Photometric System Filters

\begin{tabular}{|c|c|c|c|c|c|c|c|c|c|c|}
\hline Filter & $\begin{array}{l}\lambda_{\text {iso }}{ }^{\mathrm{a}} \\
(\mathrm{nm})\end{array}$ & $\begin{array}{c}F_{\lambda} \\
\left(\mathrm{erg} \mathrm{s}^{-1} \mathrm{~cm}^{-2} \AA^{-1}\right)\end{array}$ & $\begin{array}{l}\text { VEGA AB } \\
\text { Magnitude }\end{array}$ & $\begin{array}{l}\lambda_{m}{ }^{\mathrm{b}} \\
(\mathrm{nm})\end{array}$ & $\begin{array}{l}c v_{m}^{-1 \mathrm{c}} \\
(\mathrm{nm})\end{array}$ & $\begin{array}{l}\lambda_{\text {eff }}{ }^{\mathrm{d}} \\
(\mathrm{nm})\end{array}$ & $\sigma$ & $Q$ & $\begin{array}{c}\text { FWHM } \\
(\mathrm{nm})\end{array}$ & $\delta$ \\
\hline A366M & 373.8 & $3.354 \mathrm{e}-09$ & 0.96 & 366.4 & 366.0 & 366.1 & 0.0216 & 0.0211 & 27.9 & 186.2 \\
\hline A394M & 398.5 & $6.851 \mathrm{e}-09$ & 0.02 & 394.4 & 393.9 & 394.1 & 0.0239 & 0.0414 & 33.0 & 222.2 \\
\hline A425M & 430.9 & $6.782 \mathrm{e}-09$ & -0.13 & 425.3 & 424.9 & 424.9 & 0.0229 & 0.0419 & 34.2 & 229.8 \\
\hline A457M & 456.8 & $6.117 \mathrm{e}-09$ & -0.18 & 457.8 & 457.4 & 457.5 & 0.0209 & 0.0412 & 33.2 & 224.9 \\
\hline A491M & 500.8 & $4.726 \mathrm{e}-09$ & -0.05 & 491.7 & 491.2 & 491.3 & 0.0208 & 0.0426 & 35.6 & 241.2 \\
\hline A522M & 522.4 & $4.145 \mathrm{e}-09$ & -0.04 & 522.6 & 522.3 & 522.4 & 0.0178 & 0.0395 & 32.6 & 218.9 \\
\hline A551M & 551.0 & $3.547 \mathrm{e}-09$ & 0.01 & 551.2 & 550.9 & 551.0 & 0.0156 & 0.0385 & 29.7 & 202.1 \\
\hline A581M & 581.1 & $3.031 \mathrm{e}-09$ & 0.07 & 581.2 & 580.9 & 580.9 & 0.0161 & 0.0407 & 32.4 & 221.1 \\
\hline A613M & 613.4 & $2.573 \mathrm{e}-09$ & 0.13 & 613.6 & 613.4 & 613.4 & 0.0149 & 0.0364 & 32.0 & 216.2 \\
\hline A646M & 650.8 & $2.119 \mathrm{e}-09$ & 0.23 & 646.4 & 646.0 & 646.1 & 0.0159 & 0.0419 & 35.7 & 241.5 \\
\hline A678M & 678.1 & $1.896 \mathrm{e}-09$ & 0.24 & 678.2 & 678.0 & 678.1 & 0.0133 & 0.0355 & 31.4 & 211.9 \\
\hline A708M & 707.9 & $1.661 \mathrm{e}-09$ & 0.29 & 707.9 & 707.7 & 707.8 & 0.0135 & 0.0347 & 33.2 & 225.3 \\
\hline A739M & 739.1 & $1.453 \mathrm{e}-09$ & 0.34 & 739.3 & 739.1 & 739.2 & 0.0117 & 0.0302 & 30.4 & 203.3 \\
\hline A770M & 770.1 & $1.277 \mathrm{e}-09$ & 0.39 & 770.2 & 769.9 & 769.9 & 0.0132 & 0.0329 & 35.4 & 239.2 \\
\hline A802M & 802.4 & $1.123 \mathrm{e}-09$ & 0.44 & 802.2 & 801.9 & 802.0 & 0.0111 & 0.0263 & 31.2 & 210.5 \\
\hline A829M & 830.2 & $1.012 \mathrm{e}-09$ & 0.48 & 829.5 & 829.3 & 829.4 & 0.0103 & 0.0226 & 29.6 & 202.1 \\
\hline A861M & 861.5 & $8.943 e-10$ & 0.54 & 861.6 & 861.3 & 861.4 & 0.0121 & 0.0233 & 36.9 & 246.4 \\
\hline A892M & 884.3 & $8.703 e-10$ & 0.50 & 891.9 & 891.7 & 891.8 & 0.0095 & 0.0162 & 30.3 & 200.2 \\
\hline A921M & 936.2 & $8.197 \mathrm{e}-10$ & 0.48 & 920.9 & 920.7 & 920.8 & 0.0096 & 0.0143 & 30.8 & 207.8 \\
\hline A948M & 950.4 & $7.502 \mathrm{e}-10$ & 0.52 & 948.3 & 948.2 & 948.2 & 0.0093 & 0.0109 & 31.9 & 208.1 \\
\hline
\end{tabular}

Notes. Columns 2-4 represent the isophotal wavelengths, flux densities, and AB magnitudes of VEGA in the ALHAMBRA photometric system.

${ }^{a}$ Isophotal wavelength defined in Equation (3).

b Wavelength-weighted average.

${ }^{c}$ Inverse of frequency-weighted average.

${ }^{\mathrm{d}}$ Effective wavelength defined in Equation (6).

parameters that only depend on the photometric system, such as the wavelength-weighted average or the frequency-weighted average (written as $c v^{-1}$ )

$$
\begin{gathered}
\lambda_{\text {med }}=\frac{\int \lambda S_{\lambda} d \lambda}{\int S_{\lambda} d \lambda}, \\
v_{\text {med }}=\frac{\int \nu S_{\nu} d(\ln v)}{\int S_{\nu} d(\ln v)},
\end{gathered}
$$

or by the effective wavelength as defined by Schneider et al. (1983)

$$
\lambda_{\text {eff }}=\exp \left[\frac{\int d(\ln v) S_{v} \ln \lambda}{\int d(\ln v) S_{v}}\right],
$$

which is halfway between the wavelength-weighted average and the frequency-weighted average (Fukugita et al. 1996). The differences between the isophotal wavelength concept and these three central approaches can be seen in Golay (1974).

The root mean square (rms) fractional widths of the filters, $\sigma$, defined as

$$
\sigma=\sqrt{\frac{\int d(\ln v) S_{v}\left[\ln \left(\frac{\lambda}{\lambda_{\text {eff }}}\right)\right]^{2}}{\int d(\ln v) S_{v}}}
$$

are useful in calculating the sensitivity of the effective wavelength to spectral slope changes,

$$
\delta \lambda_{\text {eff }}=\lambda_{\text {eff }} \sigma^{2} \delta n,
$$

where $n$ is the local power-law index of the $\operatorname{SED}\left(f_{v} v^{n}\right)$, and the effective band width, $\delta$, is given by

$$
\delta=2(2 \ln 2)^{1 / 2} \sigma \lambda_{\text {eff }}
$$

We also calculate the flux sensitivity quantity $Q$, defined as

$$
Q=\int d(\ln v) S_{v},
$$

which allows a quick approximation to the response of the system to a source of known flux in the following way:

$$
N_{e}=A t Q f_{v_{\mathrm{eff}}} h^{-1},
$$

where $N_{e}$ is the number of photoelectrons collected with a system of effective area A integrating for a time $t$ on a source of flux $f_{v}$, and $h$ is the Planck constant. As an example, the number of photoelectrons collected using the ALHAMBRA photometric system, in $1 \operatorname{arcsec}^{2}$, integrating for a time of $5000 \mathrm{~s}$ on a source of $A B=23 \mathrm{mag} \operatorname{arcsec}^{-2}$ in filter $A 491 \mathrm{M}$ is $1.54 \times 10^{4}$ photoelectrons.

Table 1 shows the values of these parameters calculated for each of the filters in the ALHAMBRA photometric system.

\section{STANDARD STARS SYSTEM}

To calibrate the ALHAMBRA photometric system, we have chosen two different sets of primary standard stars. The first one is a set of 31 classic spectrophotometric standard stars from several libraries, such as Oke \& Gunn (1983), Oke (1990), Massey \& Gronwall (1990), and Stone (1996), together with the main standard stars adopted by the Hubble Space Telescope (HST; Bohlin 2007), and the SDSS standard BD + 174708 (Tucker et al. 2001). This set was chosen in order to anchor the ALHAMBRA photometric system with some standards that have been used on important photometric systems such as the SDSS, with which we have established some transformation equations, as explained in the following sections. These objects are stars with no detected variability within a few 
Table 2

ALHAMBRA AB Magnitudes of the 31 Spectrophotometric Primary Standard Stars

\begin{tabular}{lrrrrr}
\hline \hline \multicolumn{1}{c}{ Star } & A366M & A491M & A613M & A770M & A921M \\
\hline g191b2b_mod_004 & 11.04 & 11.57 & 11.99 & 12.45 & 12.82 \\
bd_75d325_stis_001 & 8.77 & 9.32 & 9.75 & 10.21 & 10.58 \\
feige34_stis_001 & 10.40 & 10.97 & 11.38 & 11.80 & 12.11 \\
p041c_stis_001 & 13.40 & 12.13 & 11.84 & 11.73 & 11.72 \\
alpha_lyr_stis_004 & 0.96 & -0.05 & 0.13 & 0.39 & 0.48 \\
\hline
\end{tabular}

(This table is available in its entirety in machine-readable and Virtual Observatory (VO) forms in the online journal. A portion is shown here for guidance regarding its form and content.)

Table 3

ALHAMBRA AB Magnitudes of the 288 Primary Standard Stars from the NGSL

\begin{tabular}{lcrrrr}
\hline \multicolumn{1}{c}{ Star } & A366M & A491M & A613M & A770M & A921M \\
\hline BD 17d4708 & 10.45 & 9.57 & 9.34 & 9.25 & 9.24 \\
Feige110 & 11.15 & 11.62 & 12.05 & 12.47 & 12.82 \\
G188-22 & 11.11 & 10.24 & 9.98 & 9.83 & 9.87 \\
HD 086986 & 9.13 & 7.94 & 8.02 & 8.14 & 8.16 \\
HD 204543 & 10.23 & 8.56 & 7.99 & 7.63 & 7.52 \\
\hline
\end{tabular}

(This table is available in its entirety in machine-readable and Virtual Observatory (VO) forms in the online journal. A portion is shown here for guidance regarding its form and content.)

millimagnitudes scale, available high-resolution spectra $(\leqslant 5 \AA)$, and flux calibrated with errors lower than $5 \%$. These standard stars are spread out all over the sky and are bright, but they do not cover all the spectral types, being mostly white dwarfs and main-sequence A-type stars.

The second set of primary standard stars is formed by 288 stars from a new spectral library, with good spectral resolution, all of them obtained and flux calibrated homogeneously, and covering a wide range of spectral types, gravities, and metallicities $\left(3440 \mathrm{~K} \leqslant T_{\text {eff }} \leqslant 44500 \mathrm{~K}, 0.45 \leqslant \log g \leqslant 4.87\right.$, and $-2 \leqslant[\mathrm{Fe} / \mathrm{H}] \leqslant 0.5)$. These spectra have been used to calibrate and calculate zero points for different photometric systems (Maíz-Apellániz 2006); they consist of 378 high signalto-noise stellar spectra, and are known as HST/STIS NGSL (Gregg et al. 2004). The wavelength range of these spectra covers from $2000 \AA$ to $10200 \AA$. These stars represent a potential set of spectrophotometric standards, being valuable in the study of atmosphere parameters using model atmosphere fluxes. Coordinates and physical properties of these stars can be found at http://archive.stsci.edu/prepds/stisngsl/.

These 288 stars together with the 31 classic spectrophotometric standards define the set of primary standards of the ALHAMBRA photometric system. AB magnitudes in the ALHAMBRA system of these stars are presented in Tables 2 and 3. Nine stars (AGK81D266, BD 17D4708, BD 28D4211, BD 75D325, FEIGE 110, FEIGE 34, G191B2B, HZ 44, P041C) are common to both sets. Magnitudes in the ALHAMBRA photometric system for these nine stars derived from the two different sources show differences lower than a few millimagnitudes over the whole filter set, indicating that both groups of standard stars are anchored to the same physical system. However, once we have established the similitude and stability of the photometric zero points for both set of standards, we will base the further analysis on the NGSL catalog, which shows a higher degree of internal homogeneity, both in wavelength and flux calibrations.
Magnitudes in the ALHAMBRA photometric system are set on the AB magnitude system (Oke \& Gunn 1983),

$$
\mathrm{AB}_{v}=-2.5 \log f_{v}-48.60
$$

where the constant comes from the expression

$$
48.6=-2.5 \log F_{0},
$$

and $F_{0}=3.63 \times 10^{-20} \mathrm{erg} \mathrm{cm}^{-2} \mathrm{~s}^{-1} \mathrm{~Hz}^{-1}$ is the flux of $V E G A$ at $\lambda=5500 \AA$ used by those authors. The parameter $f_{v}$ is the flux per unit frequency of an object in erg $\mathrm{cm}^{-2} \mathrm{~s}^{-1} \mathrm{~Hz}^{-1}$.

The AB magnitudes will be estimated as follows:

$$
\mathrm{AB}_{v}=-2.5 \log \frac{\int f_{v} S_{v} d(\log v)}{\int S_{v} d(\log v)}-48.6
$$

where $S_{v}$ is the response function of the system corresponding to the atmosphere-telescope-filter-detector combination.

Regarding the observational part of the ALHAMBRA project, the adequate definition of the photometric system must fit both the LAICA camera and its geometry. LAICA's focal plane is made up of four CCDs that partly cover a total field of $44^{\prime} .36 \times 44^{\prime} .36$, and it needs four filter sets, each one univocally associated to each of the CCDs. The standard ALHAMBRA photometric system is chosen as the one at position number 3 of the LAICA camera, and three replicas have been built to complete the observational setting. Comparison of the three instrumental replicas and the ALHAMBRA photometric system has been performed using the VEGA spectrum above. We calculated the absolute differences between the $\mathrm{AB}$ magnitudes of the star in each one of the three replicas and the standard ALHAMBRA value, detecting that filter A366M shows the maximum absolute difference (up to $0.05 \mathrm{mag}$ ). In the case of filter A394M, there is a maximum of 0.02 mag offset, filter $\mathrm{A} 425 \mathrm{M}$ has a $0.01 \mathrm{mag}$ offset, and the rest of the filters have smaller differences.

\begin{tabular}{cccccc}
\hline \hline Filter & CCD1 & CCD2 & CCD3 & CCD4 & $\Delta$ max \\
\hline A366M & 0.97 & 0.97 & 0.96 & 0.92 & 0.05 \\
A394M & 0.03 & 0.02 & 0.02 & 0.01 & 0.02 \\
A425M & -0.14 & -0.13 & -0.13 & -0.13 & 0.01 \\
\hline
\end{tabular}

We have developed a statistical analysis on the distribution of differences, using synthetic AB magnitudes of the 288 standard stars from the NGSL. Table 4 shows the result of this analysis. Once again filter A366M presents the largest difference in magnitude, reaching up to $0.0689 \mathrm{mag}$ for some stars in the sample. For the whole sample, the median of the differences in absolute value between filter A366M AB magnitudes in the ALHAMBRA photometric system and those determined from the three replicas, is $0.0174 \mathrm{mag}$, with a rms (calculated through median absolute deviation, MAD) of 0.0088 . In the other filters, the median of the absolute differences is less than $0.01 \mathrm{mag}$, becoming smaller as we move to redder wavelengths.

\section{ALHAMBRA-SDSS TRANSFORMATION EQUATIONS}

\subsection{Based on the Standard Stars}

The SDSS is a well-known astronomical project that has obtained deep multi-color images covering more than a quarter 
Table 4

Analysis of the Four Instances of the ALHAMBRA System Used with the LAICA Camera

\begin{tabular}{cccc}
\hline \hline Filter & $\Delta$ Max & Median & rms \\
\hline A366M & 0.0689 & 0.0174 & 0.0088 \\
A394M & 0.0236 & 0.0064 & 0.0053 \\
A425M & 0.0592 & 0.0058 & 0.0073 \\
A457M & 0.0148 & 0.0028 & 0.0025 \\
A491M & 0.0133 & 0.0011 & 0.0013 \\
A522M & 0.0325 & 0.0022 & 0.0021 \\
A551M & 0.0092 & 0.0008 & 0.0009 \\
A581M & 0.0110 & 0.0024 & 0.0019 \\
A613M & 0.0052 & 0.0003 & 0.0003 \\
A646M & 0.0085 & 0.0012 & 0.0007 \\
A678M & 0.0247 & 0.0009 & 0.0007 \\
A708M & 0.0031 & 0.0002 & 0.0001 \\
A739M & 0.0228 & 0.0009 & 0.0006 \\
A770M & 0.0042 & 0.0003 & 0.0003 \\
A802M & 0.0134 & 0.0006 & 0.0006 \\
A829M & 0.0016 & 0.0003 & 0.0003 \\
A861M & 0.0133 & 0.0009 & 0.0007 \\
A892M & 0.0034 & 0.0002 & 0.0003 \\
A921M & 0.0013 & 0.0003 & 0.0001 \\
A948M & 0.0028 & 0.0005 & 0.0003 \\
\hline
\end{tabular}

Notes. The first column is the list of ALHAMBRA filters; the second is the maximum absolute difference between ALHAMBRA $\mathrm{AB}$ magnitudes and the magnitudes obtained from the three filterdetector replicas at each ALHAMBRA filter; the third represents the median of the former absolute differences; the fourth is the median absolute deviation of the distribution.

of the sky. Its photometric system is formed by five nonoverlapping color bands (ugriz) that cover the complete optical range from $3000 \AA$ to $11000 \AA$ (Fukugita et al. 1996; Smith et al. 2002). Given that the SDSS and the ALHAMBRA photometric systems lay on the same reference standard stars, a set of transformation equations can be determined between both systems based on the NGSL stars.

Based on the proximity of their effective wavelengths (Table 1), we select the ALHAMBRA filters which better correspond to the five SDSS bands as

\begin{tabular}{llll}
\hline \hline$u$ & $354.0 \mathrm{~nm}$ & A366M & $366.1 \mathrm{~nm}$ \\
$g$ & $477.0 \mathrm{~nm}$ & A $491 \mathrm{M}$ & $491.3 \mathrm{~nm}$ \\
$r$ & $622.2 \mathrm{~nm}$ & A613M & $613.4 \mathrm{~nm}$ \\
$i$ & $763.2 \mathrm{~nm}$ & A770M & $769.9 \mathrm{~nm}$ \\
$z$ & $904.9 \mathrm{~nm}$ & A921M & $920.7 \mathrm{~nm}$ \\
\hline
\end{tabular}

In this way, a zero-order approximation to the SDSS photometric system could be given by the ALHAMBRA colors A366M-A491M (corresponding to the $u-g$ SDSS color), A491M-A613M ( $g-r$ color), A613M-A770M ( $r-i$ color), and A770M-A921M ( $i-z$ color $)$.

However, one has to take into account the different widths of the filters in the two systems (see Figure 1(b) for a graphic comparison). The ALHAMBRA photometric system uses mediumband filters $(\approx 31 \mathrm{~nm}$ wide), so they are more sensitive to discontinuities in the SED. On the contrary, the SDSS wideband filters transmit more energy, permitting the detection of fainter objects, but showing more difficulties in the interpretation and analysis of the information derived from their photometric measurements.
The transformation equations from SDSS magnitudes to the ALHAMBRA photometric system (and vice versa) have been calculated using synthetic AB magnitudes for the NGSL subset of the ALHAMBRA primary standards. The ugriz synthetic magnitudes were obtained from the filters and detector set, following the SDSS standard system given by Smith et al. (2002; USNO), located in the US Naval Observatory $1 \mathrm{~m}$ telescope.

The elaboration of the linear model used to obtain the magnitudes in the ALHAMBRA photometric system from SDSS photometry has been developed using the statistical software $\mathbf{R},{ }^{16}$ in particular the statistical task called "Backward Stepwise Regression" that uses the Bayesian Information Criterion (BIC), a tool for model selection defined as

$$
\mathrm{BIC}=-2 \ln L+k \ln (n) \text {, }
$$

where $L$ is the maximized value of the likelihood function for the estimated model, $k$ the number of free parameters to be estimated, and $n$ is the sample size (number of observations).

The transformation equations are, in general, valid for the range of stellar parameters covered by the selected 288 NGSL stars. Table 5 shows the coefficients of the transformation from SDSS magnitudes to ALHAMBRA photometry. The first column comprises the dependent variables of the linear regression which are composed of a color formed with an ALHAMBRA band minus an SDSS band; the independent variables are correlative SDSS colors. We calculate this type of transformation instead of directly using the AB ALHAMBRA magnitudes as the dependent variables and the SDSS magnitudes as the independent variables in order to force the sum of the linear model coefficients to be 1 , a necessary condition when we are comparing magnitudes and not colors. From a model such as

$$
\operatorname{Alh}_{i}=\sum_{j} a_{i j} \cdot \operatorname{Sloan}_{j}+b_{i},
$$

it follows that the same star observed (using any photometric system) at different distances would have the same magnitude plus a single constant equal for all filters, $\mu$, so the equations should verify

$$
\begin{aligned}
\operatorname{Alh}_{i}+\mu & =\sum_{j} a_{i j} \cdot\left(\operatorname{Sloan}_{j}+\mu\right)+b_{i} \\
& =\sum_{j} a_{i j} \cdot \operatorname{Sloan}_{j}+b_{i}+\left(\sum_{j} a_{i j}\right) \cdot \mu,
\end{aligned}
$$

which implies that the coefficients in the transformation equations should satisfy that the sum of all the coefficients is equal to 1 . With this in mind, the equations can be posed as

$$
\operatorname{Alh}_{i}-\operatorname{Sloan}_{k}=\sum_{j=1}^{4} c_{i j} \cdot\left(\operatorname{Sloan}_{j}-\operatorname{Sloan}_{j+1}\right)+d_{i}
$$

where $\operatorname{Sloan}_{k}$ is any of the five SDSS bands.

Table 6 shows the coefficients of the inverse transformation equations, from ALHAMBRA photometry to SDSS magnitudes, obtained with the same procedure.

\subsection{Redshift Galaxy Templates}

Color transformations for stars, in general, would not offer accurate results for galaxies, especially when the effects of redshift and intergalactic absorption are taken into account. It is

\footnotetext{
16 http://www.r-project.org
} 
Table 5

Coefficients of the Transformation Equations from SDSS to ALHAMBRA Photometry

\begin{tabular}{|c|c|c|c|c|c|c|}
\hline \multicolumn{6}{|c|}{ Synthetic } & \multirow[t]{2}{*}{ Error } \\
\hline & $\emptyset$ & $u-g$ & $g-r$ & $r-i$ & $i-z$ & \\
\hline A366M- $i$ & -0.0209 & 0.9813 & 0.7946 & 1.0889 & & 0.038 \\
\hline A394M- $i$ & -0.1997 & 0.4183 & 1.9673 & 0.8166 & -0.8552 & 0.097 \\
\hline $\mathrm{A} 425 \mathrm{M}-i$ & -0.0639 & 0.1475 & 1.4885 & 0.8921 & & 0.058 \\
\hline $\mathrm{A} 457 \mathrm{M}-i$ & -0.0094 & & 1.0195 & 1.0358 & 0.1799 & 0.037 \\
\hline A491M- $i$ & 0.0240 & & 0.6274 & 1.2114 & 0.1969 & 0.024 \\
\hline $\mathrm{A} 522 \mathrm{M}-i$ & -0.0239 & & 0.6204 & 1.0030 & & 0.028 \\
\hline A551M- $g$ & -0.0093 & -0.0108 & -0.6518 & 0.1231 & -0.0975 & 0.010 \\
\hline A581M-g & -0.0029 & -0.0018 & -0.9166 & 0.2327 & -0.0547 & 0.007 \\
\hline A613M-g & -0.0009 & 0.0129 & -1.0759 & 0.1896 & & 0.009 \\
\hline A646M- $g$ & -0.0023 & 0.0159 & -1.0349 & -0.2544 & & 0.011 \\
\hline A678M-g & 0.0131 & 0.0077 & -1.2133 & -0.1184 & 0.1035 & 0.022 \\
\hline A708M-g & -0.0155 & 0.0218 & -1.1169 & -0.4915 & & 0.014 \\
\hline A739M- $g$ & -0.0096 & -0.0104 & -0.9076 & -1.1087 & 0.0677 & 0.006 \\
\hline A770M-g & 0.0165 & -0.0068 & -1.0636 & -0.9769 & & 0.007 \\
\hline A802M-g & -0.0005 & 0.0139 & -1.0063 & -1.2263 & -0.1022 & 0.006 \\
\hline A $829 \mathrm{M}-g$ & 0.0036 & 0.0199 & -1.0274 & -1.2496 & -0.2488 & 0.006 \\
\hline A861M-g & 0.0159 & 0.0211 & -1.0723 & -1.0679 & -0.6102 & 0.011 \\
\hline A892M-g & 0.0171 & -0.0192 & -1.0259 & -1.0268 & -0.8638 & 0.015 \\
\hline A921M-g & 0.0055 & -0.0163 & -0.9414 & -1.0725 & -1.1318 & 0.019 \\
\hline A948M-g & 0.0221 & & -0.9802 & -0.9667 & -1.4006 & 0.021 \\
\hline
\end{tabular}

Note. Last column represents the residual standard error of each fit.

Table 6

Coefficients of the Transformation Equations from ALHAMBRA to SDSS Photometry

\begin{tabular}{|c|c|c|c|c|c|}
\hline \multicolumn{6}{|c|}{ Synthetic } \\
\hline & $u-\mathrm{A} 522 \mathrm{M}$ & $g-\mathrm{A} 613 \mathrm{M}$ & $r-\mathrm{A} 522 \mathrm{M}$ & $i$-A522M & $z-\mathrm{A} 613 \mathrm{M}$ \\
\hline$\varnothing$ & -0.0247 & 0.0197 & -0.0006 & -0.0002 & -0.0116 \\
\hline A366M-A394M & 1.0741 & & & & \\
\hline A394M-A425M & 1.2574 & 0.0467 & & -0.0142 & -0.0337 \\
\hline A425M-A457M & 0.9768 & 0.2250 & & 0.0177 & \\
\hline A457M-A491M & 1.4529 & 0.2282 & -0.0535 & -0.0336 & \\
\hline A491M-A522M & 0.8850 & 0.4580 & & & \\
\hline A522M-A551M & & 0.6206 & -1.0334 & -1.0117 & \\
\hline A551M-A581M & & 1.4129 & -0.8928 & -0.9767 & \\
\hline A581M-A613M & & 1.3538 & -0.5793 & -0.9498 & \\
\hline A613M-A646M & & & -0.3299 & -0.9424 & -0.9459 \\
\hline A646M-A678M & -0.2832 & & -0.1895 & -0.9525 & -0.9399 \\
\hline A678M-A708M & 1.9317 & & & -1.0060 & -1.0696 \\
\hline A708M-A739M & -1.6333 & -0.4194 & -0.2017 & -0.8624 & -1.0935 \\
\hline A739M-A770M & -1.7633 & & -0.1224 & -0.5364 & -0.7336 \\
\hline A770M-A $802 \mathrm{M}$ & & 0.6199 & & -0.2679 & -1.0201 \\
\hline A $802 M-A 829 M$ & 2.5577 & 1.2026 & 0.3267 & & -0.5209 \\
\hline A829M-A861M & & -0.2826 & & & -1.0507 \\
\hline A861M-A892M & & & & & -0.5793 \\
\hline A892M-A921M & -1.2739 & -0.2896 & -0.0429 & & \\
\hline A921M-A948M & & & & & -0.7648 \\
\hline Error & 0.0253 & 0.0121 & 0.0035 & 0.0024 & 0.0102 \\
\hline
\end{tabular}

Notes. For each SDSS filter minus one of the ALHAMBRA filters, the column below shows the coefficients of each one of the 19 ALHAMBRA colors, plus the independent term. Last row gives the residual standard error of each fit.

therefore desirable to describe the colors of observed galaxies at different redshifts in the system as accurately as possible. To do so, we use the empirically calibrated library of Benítez et al. (2004). We generate estimated colors for the galaxies in the library in redshift intervals of 0.05 , using the SDSS filter $r$ as reference for all the ALHAMBRA medium-band filters. All colors correspond to the AB system, and were calculated using the functions included in the module bpz_tools from the
BPZ package (Benítez 2000) which offers an accuracy similar to that of SYNPHOT (Baggett et al. 1997). We present here the tables for the E/S0, Sbc, Im, SB2, SB3, and Scd templates for the ALHAMBRA photometric system (Tables 7-12). These values allow us to determine in an easy way the $k$-correction which arises from estimating spectral indices at observing frequencies other than the rest frequency. As an example, Figures 2 and 3 present the variation of the 
Table 7

Multi-instrument Colors of the E/S0 Galaxy Template from Benítez et al. (2004) in the ALHAMBRA System (AB Magnitude) and SDSS $r$ (AB Magnitude)

\begin{tabular}{cccccc}
\hline \hline$z$ & A366M- $r$ & A491M-r & A613M- $r$ & A770M- $r$ & A921M- $r$ \\
\hline 0.00 & 2.26 & 0.55 & -0.01 & -0.41 & -0.66 \\
0.25 & 3.00 & 1.32 & -0.01 & -0.58 & -0.94 \\
1.00 & 3.47 & 2.26 & 0.12 & -0.88 & -2.38 \\
1.50 & 1.69 & 1.06 & 0.21 & -1.98 & -3.01 \\
2.25 & 1.17 & 0.47 & 0.09 & -0.83 & -2.48 \\
\hline
\end{tabular}

(This table is available in its entirety in machine-readable and Virtual Observatory (VO) forms in the online journal. A portion is shown here for guidance regarding its form and content.)

Table 8

Multi-instrument Colors of the Sbc Galaxy Template from Benítez et al. (2004) in the ALHAMBRA System (AB Magnitude) and SDSS $r$ (AB Magnitude)

\begin{tabular}{cccccc}
\hline \hline$z$ & A366M- $r$ & A491M-r & A613M- $r$ & A770M- $r$ & A921M-r \\
\hline 0.00 & 1.64 & 0.45 & 0.01 & -0.33 & -0.55 \\
0.25 & 2.28 & 0.76 & 0.00 & -0.44 & -0.73 \\
1.00 & 1.03 & 0.56 & 0.07 & -1.17 & -1.94 \\
1.50 & 1.22 & 0.34 & 0.01 & -0.49 & -1.46 \\
2.25 & 1.92 & 0.69 & -0.01 & -0.34 & -0.67 \\
\hline
\end{tabular}

(This table is available in its entirety in machine-readable and Virtual Observatory (VO) forms in the online journal. A portion is shown here for guidance regarding its form and content.)

Table 9

Multi-instrument Colors of the Im Galaxy Template from Benítez et al. (2004) in the ALHAMBRA System (AB Magnitude) and SDSS $r$ (AB Magnitude)

\begin{tabular}{cccccc}
\hline \hline$z$ & A366M-r & A491M-r & A613M-r & A770M- $r$ & A921M-r \\
\hline 0.00 & 0.75 & -0.00 & -0.00 & -0.19 & -0.30 \\
0.25 & 1.20 & 0.43 & -0.17 & -0.13 & -0.28 \\
1.00 & 0.27 & 0.15 & 0.02 & -0.63 & -1.00 \\
1.50 & 0.42 & 0.07 & 0.00 & -0.13 & -0.76 \\
2.25 & 0.95 & 0.27 & -0.01 & -0.09 & -0.16 \\
\hline
\end{tabular}

(This table is available in its entirety in machine-readable and Virtual Observatory (VO) forms in the online journal. A portion is shown here for guidance regarding its form and content.)

Table 10

Multi-instrument Colors of the SB2 Galaxy Template from Benítez et al. (2004) in the ALHAMBRA System (AB Magnitude) and SDSS $r$ (AB Magnitude)

\begin{tabular}{cccccr}
\hline \hline$z$ & A366M- $r$ & A491M-r & A613M-r & A770M- $r$ & A921M-r \\
\hline 0.00 & 0.54 & -0.34 & 0.24 & -0.02 & -0.25 \\
0.25 & 1.00 & 0.44 & -0.51 & 0.21 & 0.01 \\
1.00 & 0.13 & 0.08 & 0.08 & -0.45 & -0.62 \\
1.50 & 0.06 & 0.12 & -0.06 & -0.02 & -0.71 \\
2.25 & 0.56 & 0.01 & -0.00 & -0.03 & -0.06 \\
\hline
\end{tabular}

(This table is available in its entirety in machine-readable and Virtual Observatory (VO) forms in the online journal. A portion is shown here for guidance regarding its form and content.)

$k$-correction for two ALHAMBRA colors, A491M-A613M and A770M-A921M, respectively, versus different values of $z$ for these three galaxy templates.

These tables must be used with caution since it is obvious that a few templates cannot represent all the possible variations of real galaxy spectra. Nevertheless, they also serve as a guide to transform galaxy colors between different photometric systems
Table 11

Multi-instrument Colors of the SB3 Galaxy Template from Benítez et al. (2004) in the ALHAMBRA System (AB Magnitude) and SDSS $r$ (AB

Magnitude)

\begin{tabular}{cccccc}
\hline \hline$z$ & A366M- $r$ & A491M- $r$ & A613M- $r$ & A770M- $r$ & A921M- $r$ \\
\hline 0.00 & 1.15 & 0.32 & 0.09 & -0.11 & -0.45 \\
0.25 & 1.05 & 0.49 & -0.04 & -0.27 & -0.40 \\
1.00 & 0.36 & 0.21 & -0.02 & -0.42 & -0.96 \\
1.50 & 0.31 & 0.20 & -0.02 & -0.24 & -0.50 \\
2.25 & 0.74 & 0.22 & -0.00 & -0.11 & -0.26 \\
\hline
\end{tabular}

(This table is available in its entirety in machine-readable and Virtual Observatory (VO) forms in the online journal. A portion is shown here for guidance regarding its form and content.)

Table 12

Multi-instrument Colors of the Scd Galaxy Template from Benítez et al. (2004) in the ALHAMBRA System (AB Magnitude) and SDSS $r$ (AB Magnitude)

\begin{tabular}{cccccc}
\hline \hline$z$ & A366M- $r$ & A491M- $r$ & A613M- $r$ & A770M- $r$ & A921M-r \\
\hline 0.00 & 1.31 & 0.30 & 0.00 & -0.22 & -0.27 \\
0.25 & 1.60 & 0.62 & -0.05 & -0.34 & -0.53 \\
1.00 & 0.61 & 0.40 & 0.01 & -0.73 & -1.35 \\
1.50 & 0.74 & 0.13 & 0.01 & -0.39 & -0.94 \\
2.25 & 1.41 & 0.48 & -0.01 & -0.15 & -0.38 \\
\hline
\end{tabular}

(This table is available in its entirety in machine-readable and Virtual Observatory (VO) forms in the online journal. A portion is shown here for guidance regarding its form and content.)

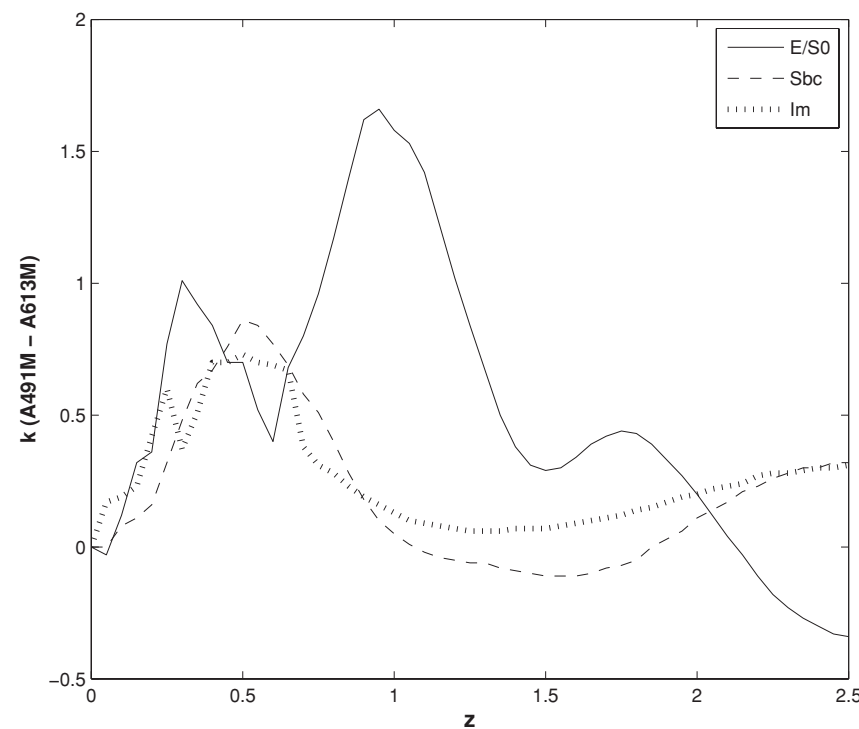

Figure 2. $k$-correction for different values of redshift $(z)$ for ALHAMBRA color A491M-A613M and three galaxy templates.

for a given spectral type and redshift. To this end, we need to find the closest possible match in redshift and spectral type in the electronic tables, using as many colors as possible. Then we look up the corresponding color in the table corresponding to the spectral type and filter of interest, using interpolation between spectral types and redshifts if needed.

\subsection{Validity of the Transformation Equations on Galaxies}

Although the transformation equations presented in Section 4.1 are calculated to be applied to stellar objects, we have carried out a quick test to see how they work for galaxy 


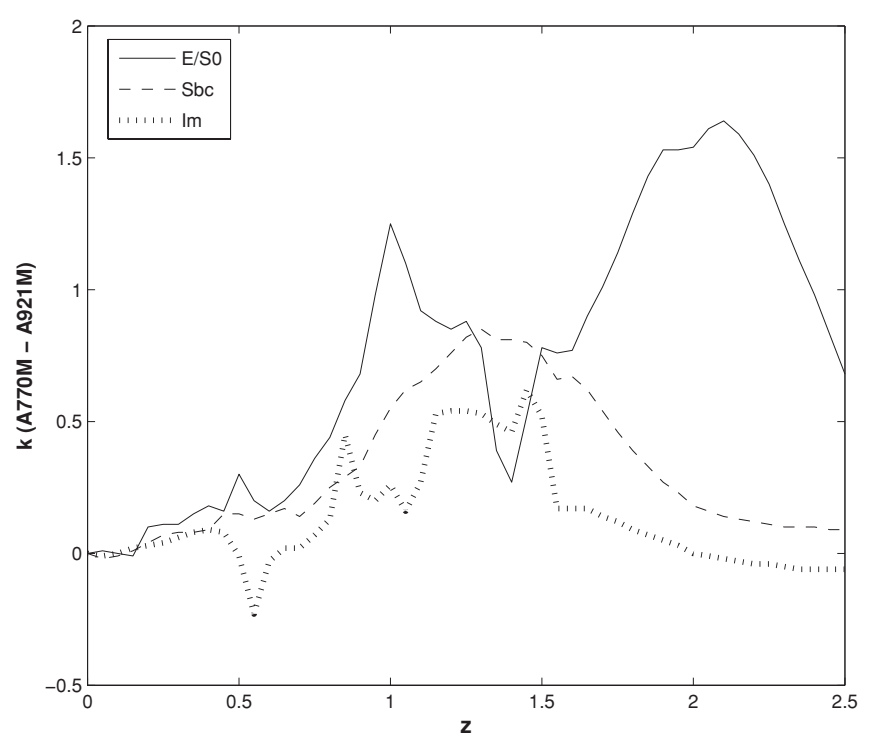

Figure 3. $k$-correction for different values of redshift $(z)$ for ALHAMBRA color A770M-A921M and three galaxy templates.

templates at $z=0$. Using Tables 7-9, we have obtained ALHAMBRA colors of the three galaxy templates: E/S0, Sbc, and Im. Applying the transformation equations to their ALHAMBRA colors, we obtained SDSS colors, and these were compared with two different results presented by Fukugita et al. (1995), where they obtained synthetic colors from the con- volution of Kennicutt's (1992) spectrophotometric atlas with the response functions of the SDSS photometric system, and Shimasaku et al. (2001), where SDSS colors of a sample of observed galaxies are presented. As Figure 4 shows, the values obtained by the ALHAMBRA-SDSS transformation equations are within the observed range of SDSS galaxy colors, taking into account the typical uncertainty in the colors that define a class of morphological type.

The validity of these transformation equations for galaxies depends on the proximity of their SEDs to the SED of the stars used in the elaboration of the equations, in our case, the 288 stars from the NGSL. Thus, in a color-color diagram, the smaller the distance of the galaxy to the distribution of the stars, the higher the confidence level will be when applying the transformation equations to this galaxy. Figure 5 shows two ALHAMBRA color-color diagrams where the same previous three galaxy templates are presented at different redshift values. E/SO and Sbc galaxies at $z=0$ are quite close to the stellar distribution, while $\operatorname{Im}$ galaxies at $z=0$ are too blue, and slightly offset from the distribution of the stellar colors.

\section{ZERO-POINT DETERMINATION}

The zero-point calibration of the observed fields in the ALHAMBRA survey has been carefully considered until we found a strategy which is robust and general enough to be applied to the whole survey and to all kind of astronomical objects. We tackled the issue in different ways, reaching a strategy with

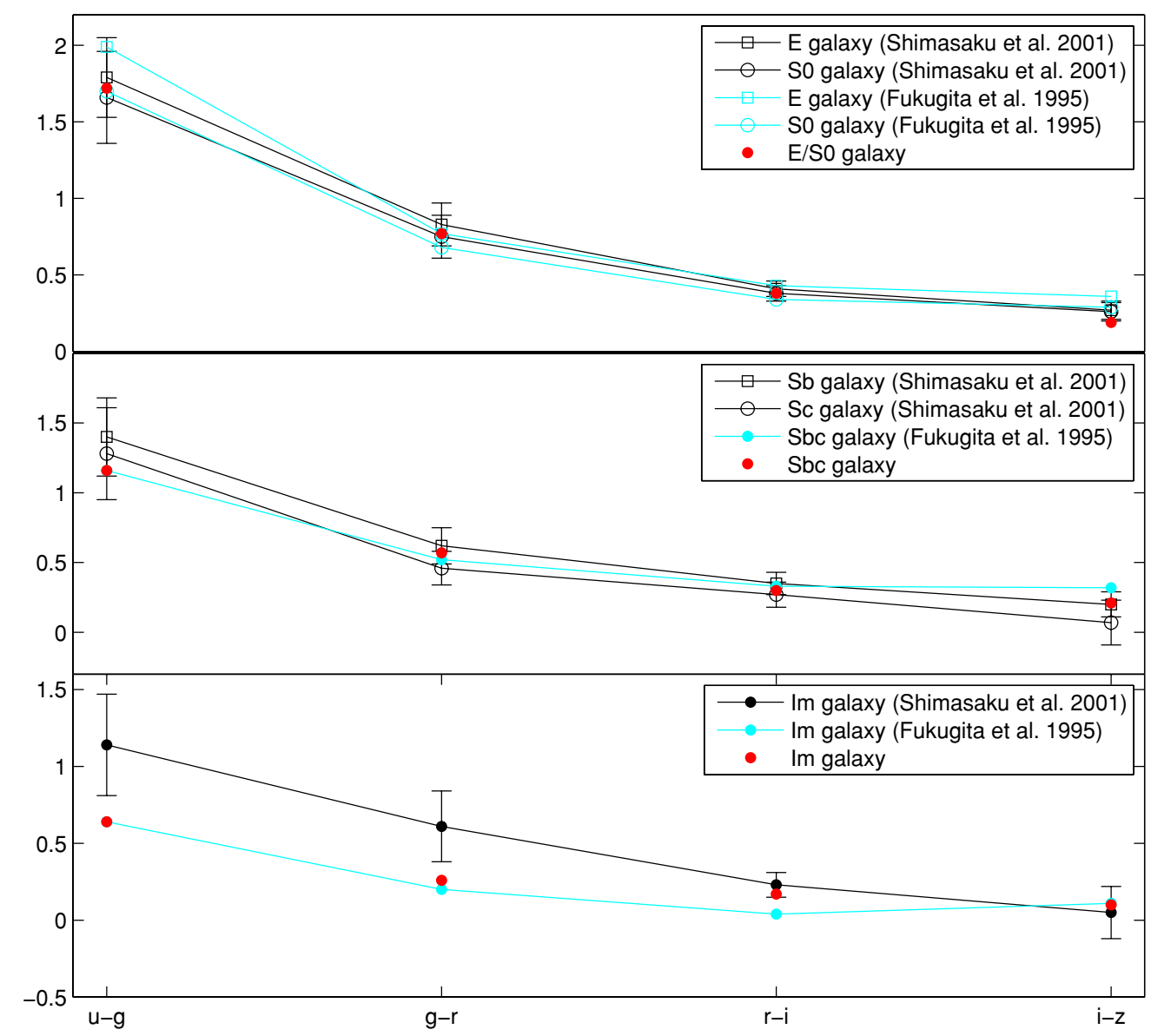

Figure 4. SDSS colors for three galaxies of different spectromorphological types. Black symbols are colors obtained from Shimasaku et al. (2001) with a rms error bar, blue ones are obtained from Fukugita et al. (1995), and red points are the colors obtained by applying the transformation equations of Section 4.1 to the ALHAMBRA photometry of these three galaxy templates (Benítez et al. 2004). 

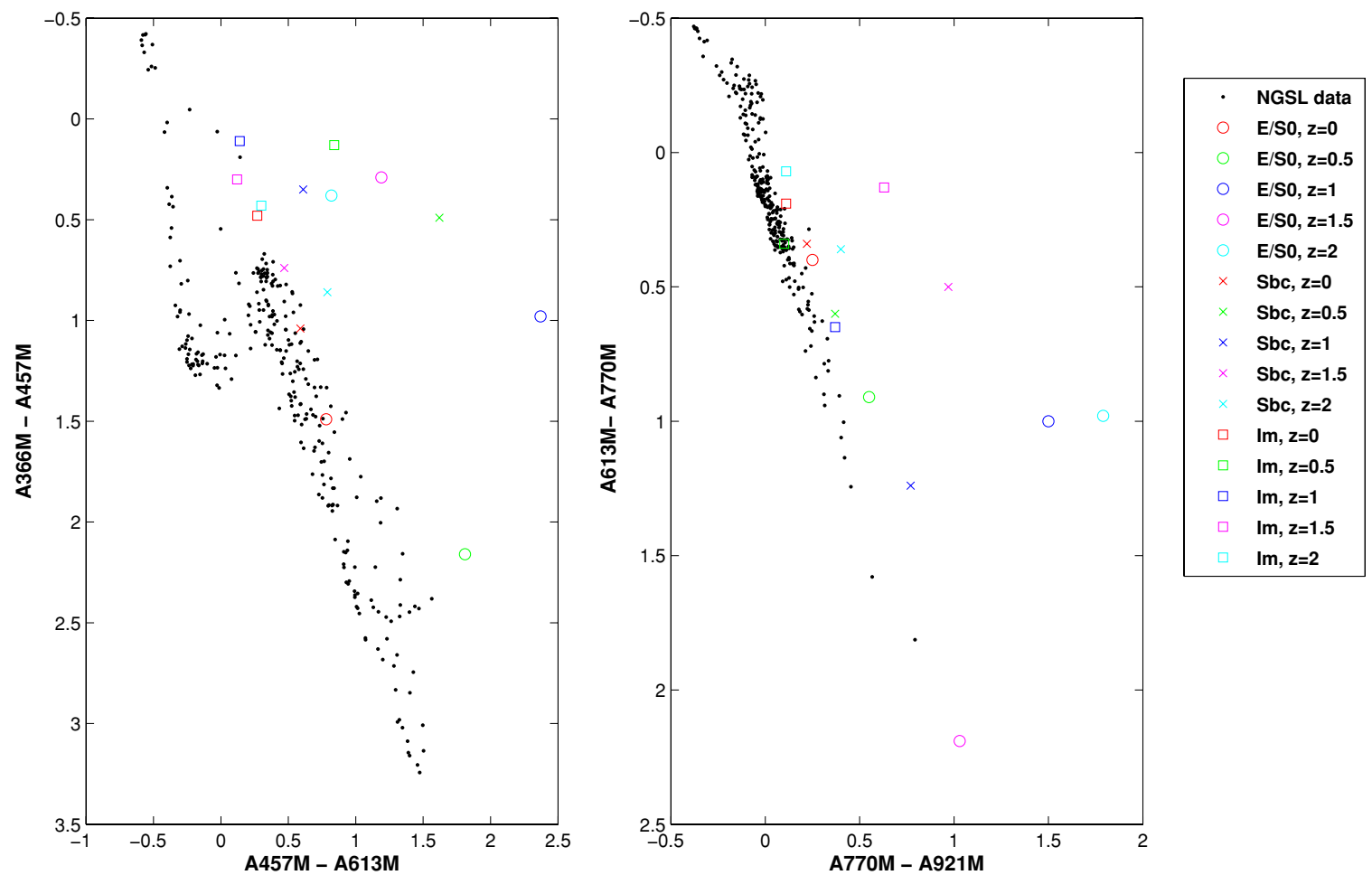

Figure 5. ALHAMBRA color-color diagrams. Black points are the set of primary standard stars from the NGSL. Circles represent E/S0 galaxy templates, crosses are Sbc galaxy templates, and squares represent Im galaxy templates, all of them from Benítez et al. (2004). Different colors show different values of $z$.

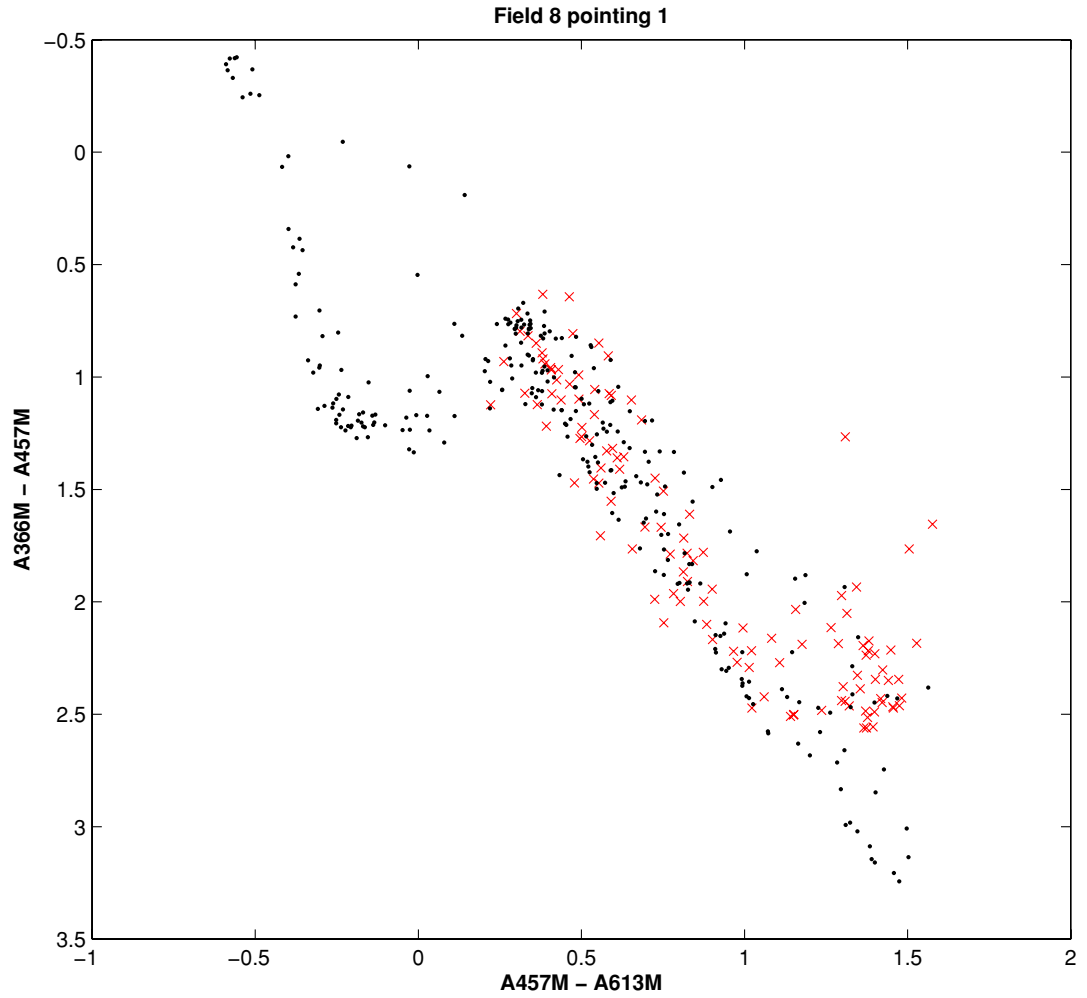

Figure 6. ALHAMBRA color-color diagram. Black points represent the 288 stars from the NGSL, and red crosses represent the field stars in field 8 , pointing 1 of the ALHAMBRA survey.

which zero points of ALHAMBRA fields are determined to an error below a few hundredths of a magnitude for the whole range of spectral types in the sample. This method entails the following steps.
1. The first task is to perform an exhaustive selection of field stars found in the ALHAMBRA source catalog for each field, selecting stellar objects with low photometric error (less than $0.15 \mathrm{mag}$ ), morphologically classified by 


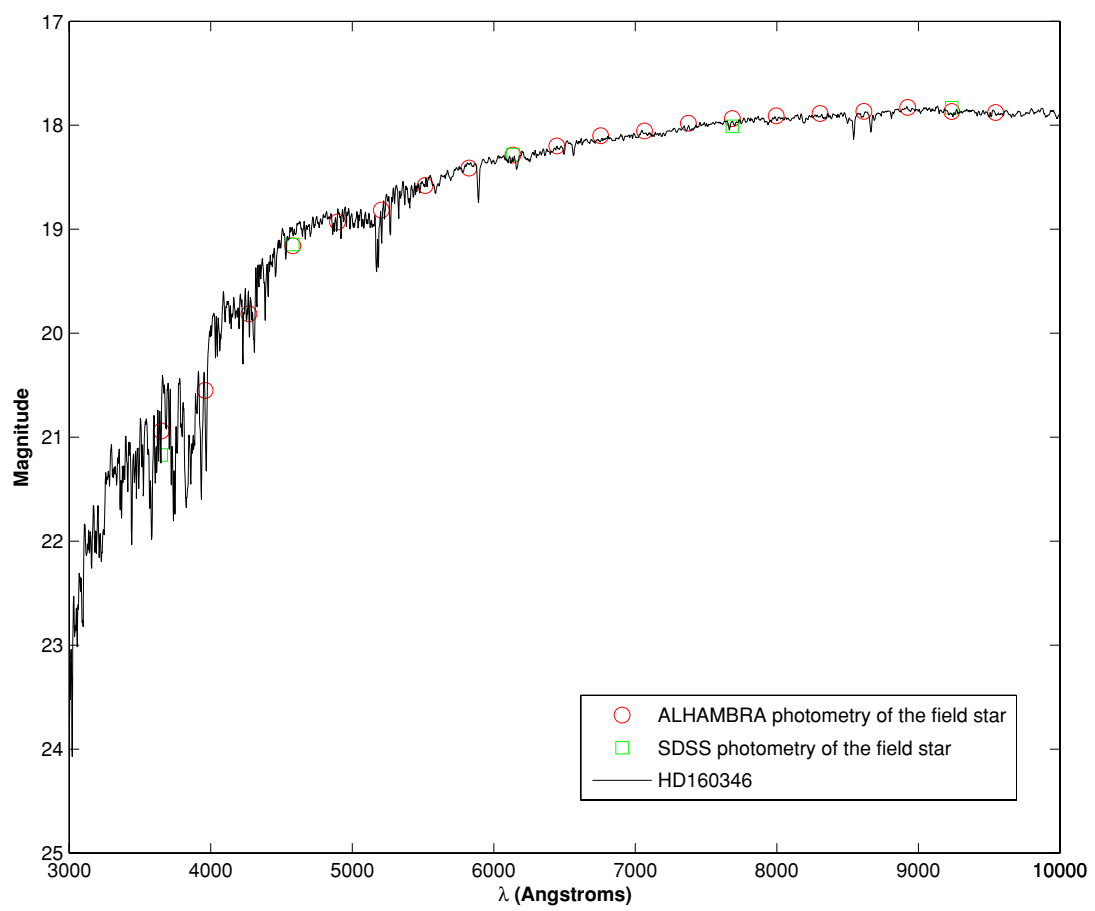

Figure 7. Spectrum in AB magnitudes of the star HD 160346, belonging to the set of NGSL standard stars. The spectrum is scaled so that this object is the best fit for the zero-point determination to the star at R.A. $=356.3323$ and decl. $=15.5486$. Red circles are the instrumental magnitudes of the field star, plus the zero points determined with our final strategy, and green squares are its SDSS photometry.

SExtractor (Bertin \& Arnouts 1996) as stellar objects at the three reddest filters, A892M, A921M, and A948M. It is possible that the pipeline may recognize the nucleus of a distant galaxy as a star in the bluest filters, so we focus primarily on classification in the reddest filters. As we show in Figure 6, the great majority of these stars are mainsequence stars of late spectral types.

2. Using this set of stars, we look for the ones that have SDSS photometry. For this task, the SDSS DR7 was used. We utilize the SkyServer Tools from its Web site. ${ }^{17}$ Among the different types of search requests we choose the more complete SQL Search, where one can search for the needed objects directly from the DR7 database.

3. Then, for each stellar object, we find the star (within the 288 NGSL objects) that best fits its SDSS photometry. To do this, we select the NGSL star that minimizes the variance of the differences between the SDSS (model) magnitudes of the NGSL star and the SDSS (observed) magnitudes of the field star. We only allow for a constant term, equal for every magnitude, related to the distance module of both stars $\left(\delta v=5 \log \left(\frac{r_{2}}{r_{1}}\right)\right)$. Once we have the NGSL object that best fit with each field star, we assign to it the expected ALHAMBRA AB magnitudes that correspond, using the already known system responses. In Figure 7, we show as an example, the spectrum of the star HD 160346 in $\mathrm{AB}$ magnitudes together with the instrumental magnitudes plus the zero points of a field star fitted with this NGSL star in the algorithm, and also its SDSS photometry.

4. Finally, the data are refined from outliers with a Chebyshev filter:

$$
\left|x_{i}-\bar{x}\right|<k * \sigma,
$$

where $\bar{x}$ is the central value of the differences, $k$ is a constant, in particular we take $k=3$, and $\sigma$ is the MAD of

\footnotetext{
17 http://cas.sdss.org/dr7/en/tools/search/radial.asp
}

Table 13

Photometric Zero Points for ALHAMBRA Field 8, Pointing 1

\begin{tabular}{ccc}
\hline \hline Filter & Zero Point & Error \\
\hline A366M & 7.4229 & 0.0011 \\
A394M & 8.3063 & 0.0008 \\
A425M & 8.4857 & 0.0007 \\
A457M & 8.6686 & 0.0009 \\
A491M & 8.7333 & 0.0004 \\
A522M & 8.5281 & 0.0005 \\
A551M & 8.5879 & 0.0004 \\
A581M & 8.6698 & 0.0006 \\
A613M & 8.4729 & 0.0004 \\
A646M & 8.5864 & 0.0004 \\
A678M & 8.5385 & 0.0005 \\
A708M & 8.3790 & 0.0004 \\
A739M & 8.8394 & 0.0004 \\
A770M & 7.9635 & 0.0003 \\
A802M & 8.4134 & 0.0004 \\
A829M & 8.4139 & 0.0005 \\
A861M & 8.1149 & 0.0004 \\
A892M & 7.2116 & 0.0004 \\
A921M & 6.5880 & 0.0006 \\
A948M & 5.9750 & 0.0006
\end{tabular}

the data. The zero point is determined as the central value of the distribution of differences between instrumental magnitudes and synthetic $\mathrm{AB}$ magnitudes.

Table 13 shows the main characteristics of the photometric zero points of the ALHAMBRA field 8, pointing 1, as an example of the results obtained when applying this strategy.

In principle, the transformation equations between SDSS and ALHAMBRA photometry and the subsequent comparison between the instrumental values and the ALHAMBRA standard values for the stars with photometry in both systems, should yield the same results. However, we noticed some systematic structure in the distribution of differences (in the sense of 


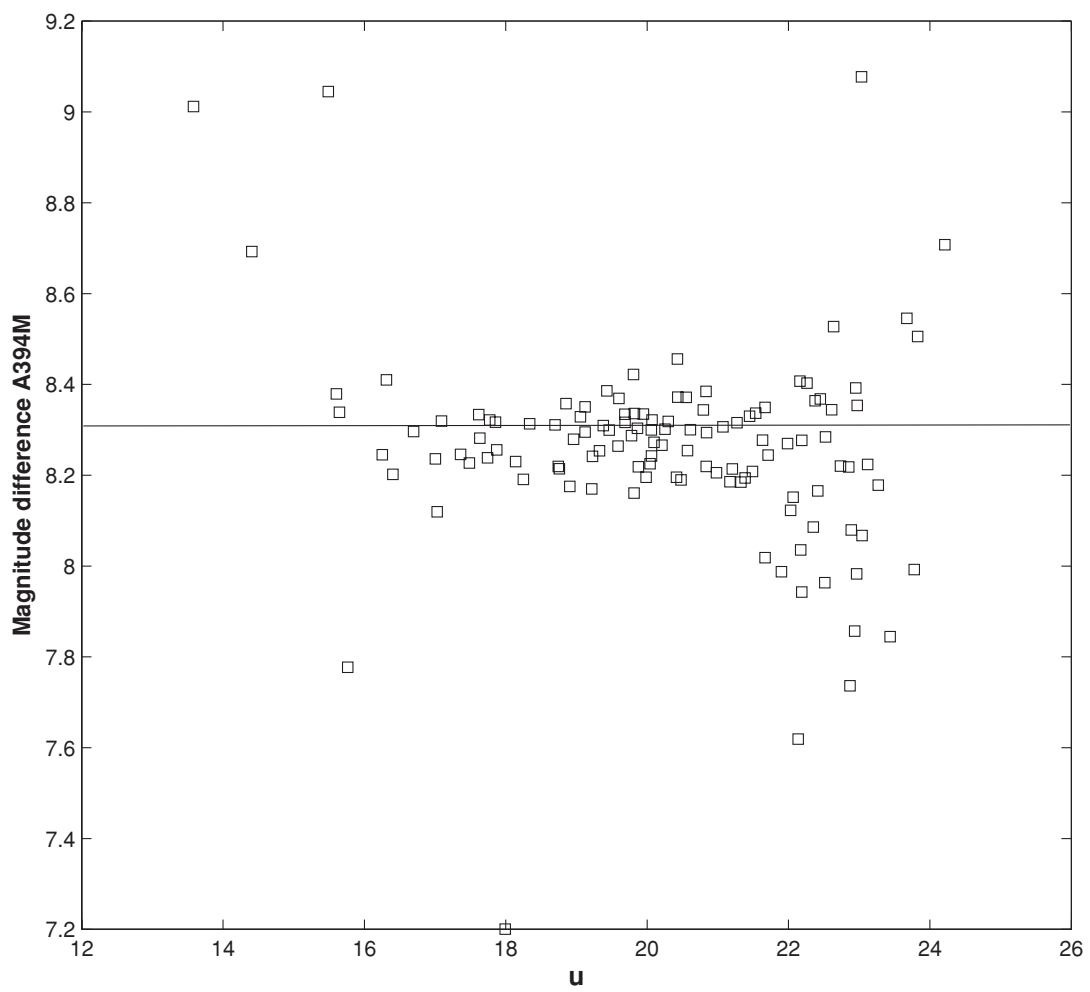

Figure 8. Magnitude differences in filter A394M vs. SDSS magnitude $u$, for the field stars in the ALHAMBRA field 8, pointing 1. They show the difference between the instrumental magnitude of the stars and the magnitude obtained from the ALHAMBRA-SDSS transformation equations. The horizontal line represents the zero-point solution determined with our chosen methodology.

instrumental minus transformed $\mathrm{AB}$ magnitude), which depends on the magnitude of the stars. The differences of the brighter stars are higher than the weaker ones, up to 1 mag ahead in some filters. In Figure 8, we plot the magnitude differences for filter A394M versus the SDSS magnitude $u$, for the same sample of field stars (ALHAMBRA field 8, pointing 1) used with the method presented above. We can see that the zero point estimated with the comparison of spectra and SEDs (see Table 13) is a good fit to the point distribution in general, although also some of the stars with brighter $u$ magnitude move systematically away from the central value. This anomalous result appears also when other photometric systems (e.g., $U B V R I)$ are compared to the SDSS system, and these transformations are then used to determine the zero point in the UBVRI system from data of the SDSS survey (as shown in Chonis \& Gaskell 2008). Those authors proposed some possible explanations to account for the observed systematic difference. We consider that a detailed discussion on the precision and accuracy of the brighter star photometry in the SDSS catalog is out of the scope of this paper. Hence, we only notice that our chosen methodology overcomes the troubles derived from a direct comparison between instrumental and standard ALHAMBRA values, obtained from SDSS-to-ALHAMBRA transformations.

\section{CONCLUSIONS AND SUMMARY}

The ALHAMBRA survey is a new extragalactic survey developed with a clear scientific objective: looking for the optimization of some variables to perform a kind of cosmic tomography of a portion of the universe. It is based on a new photometric system, consisting of 20 filters covering all the optical spectral range, and the three classical $J H K_{s}$ NIR bands. The survey camera for the optical range is LAICA, installed on the prime focus of the $3.5 \mathrm{~m}$ telescope of the Calar Alto Astronomical Observatory. The geometry of this instrument, with four different CCDs, is important to understand the ALHAMBRA observational strategy.

We present the characterization of the ALHAMBRA photometric system as defined by the product of three different response functions: detector, filters, and atmosphere.

The set of primary standard stars, which defines the ALHAMBRA photometric system, is formed by 31 classic spectrophotometric standard stars from several libraries together with 288 stars from the NGSL, which cover a wide range of spectral types and metallicities. Since the ALHAMBRA photometric system observes with four sets of filter+detector combinations (associated to each of the LAICA detectors), we have chosen one of the sets as the definition of the system, and developed a statistical analysis of the differences between the $\mathrm{AB}$ magnitudes in each one of the others. This analysis shows that the maximum difference between magnitudes, takes place in the bluest filter, A366M, with a median difference of $0.0174 \mathrm{mag}$, being much smaller in the others 19 filters.

Transformation equations from SDSS photometry to the ALHAMBRA photometric system (and vice versa) have been elaborated using synthetic magnitudes of the 288 standard stars from the NGSL, making use of "Backward Stepwise Regression," and the "BIC" as tool for model selection. We have also shown some examples of galaxy colors and their redshift evolution in our system. In particular, we show the ALHAMBRA colors for three galaxy templates $(\mathrm{E} / \mathrm{S} 0$, Sbc, and $\mathrm{Im}$ ) at different redshifts, from $z=0.00$ to $z=2.50$, with a step of 0.05 in $z$.

The strategy of the observational determination of the photometric zero point has been worked out in detail, with special 
attention to the problems that appear when these zero points are calculated based on the SDSS transformation equations. These problems are also present on other photometric systems different to our own (e.g., $U B V R I$ ). The method shown in this paper overcomes these trouble, allowing us to determine the zero point of any ALHAMBRA field with an error below few hundredths of magnitude.

We thank Jesùs Maíz-Apellániz for providing data and advice. This publication makes use of data from the SDSS. Funding for the SDSS and SDSS-II has been provided by the Alfred P. Sloan Foundation, the Participating Institutions, the National Science Foundation, the U.S. Department of Energy, the National Aeronautics and Space Administration, the Japanese Monbukagakusho, the Max Planck Society, and the Higher Education Funding Council for England. The SDSS Web site is http://www.sdss.org/. The SDSS is managed by the Astrophysical Research Consortium for the Participating Institutions. The Participating Institutions are the American Museum of Natural History, Astrophysical Institute Potsdam, University of Basel, University of Cambridge, Case Western Reserve University, University of Chicago, Drexel University, Fermilab, the Institute for Advanced Study, the Japan Participation Group, Johns Hopkins University, the Joint Institute for Nuclear Astrophysics, the Kavli Institute for Particle Astrophysics and Cosmology, the Korean Scientist Group, the Chinese Academy of Sciences (LAMOST), Los Alamos National Laboratory, the Max-Planck-Institute for Astronomy (MPIA), the Max-Planck-Institute for Astrophysics (MPA), New Mexico State University, Ohio State University, University of Pittsburgh, University of Portsmouth, Princeton University, the United States Naval Observatory, and the University of Washington.

Some/all of the data presented in this paper were obtained from the Multimission Archive at the Space Telescope Science Institute (MAST). STScI is operated by the Association of Universities for Research in Astronomy, Inc., under NASA contract NAS5-26555. Support for MAST for non-HST data is provided by the NASA Office of Space Science via grant NAG5-7584 and by other grants and contracts.

We acknowledge support from the Spanish Ministerio de Educación y Ciencia through grant AYA2006-14056 BES-200714764. E.J.A. acknowledges the financial support from the Spanish MICINN under the Consolider-Ingenio 2010 Program grant CSD2006-00070: First Science with the GTC.

\section{REFERENCES}

Baggett, S., Casertano, S., Gonzaga, S., \& Ritchie, C. 1997, Instrument Science Report (WFCP2 97-10; Baltimore, MD: STScI)

Benítez, N. 2000, ApJ, 536, 571

Benítez, N., et al. 2004, ApJS, 150, 1

Benítez, N., et al. 2009, ApJ, 692, L5

Bertin, E., \& Arnouts, S. 1996, A\&AS, 117, 393

Bohlin, R. C. 2007, in ASP Conf. Ser. 364, The Future of Photometric, Spectrophotometric and Polarimetric Standardization, ed. C. Sterken (San Francisco, CA: ASP), 315

Chonis, T. S., \& Gaskell, C. M. 2008, AJ, 135, 264

Fukugita, M., Ichikawa, T., Gunn, J. E., Doi, M., Shimasaku, K., \& Schneider, D. P. 1996, AJ, 111, 1748

Fukugita, M., Shimasaku, K., \& Ichikawa, T. 1995, PASP, 107, 945

Golay, M. 1974, in Astrophys. Space Sci. Libr., Vol. 41, Introduction to Astronomical Photometry (Dordrecht: Reidel), 375

Gregg, M. D., et al. 2004, BAAS, 36, 1496

Kennicutt, R. C., Jr. 1992, ApJS, 79, 255

Kurucz, R. L. 2005, Mem. Soc. Astron. Ital. Suppl., 8, 14

Maíz-Apellániz, J. 2006, AJ, 131, 1184

Massey, P., \& Gronwall, C. 1990, ApJ, 358, 344

Moles, M., et al. 2008, AJ, 136, 1325

Oke, J. B. 1990, AJ, 99, 1621

Oke, J. B., \& Gunn, J. E. 1983, ApJ, 266, 713

Sánchez, S. F., Aceituno, J., Thiele, U., Pérez-Ramírez, D., \& Alves, J. 2007, PASP, 119, 1186

Schneider, D. P., Gunn, J. E., \& Hoessel, J. G. 1983, ApJ, 264, 337

Shimasaku, K., et al. 2001, AJ, 122, 1238

Smith, J. A., et al. 2002, AJ, 123, 2121

Stone, R. P. S. 1996, ApJS, 107, 423

Tokunaga, A. T., \& Vacca, W. D. 2005, PASP, 117, 1459

Tucker, D. L., Smith, J. A., \& Brinkmann, J. 2001, in ASP Conf. Ser. 232, The New Era of Wide Field Astronomy, ed. R. Clowes, A. Adamson, \& G. Bromage (San Francisco, CA: ASP), 13 